\title{
EFFICACY OF SERPENTINE-TYPE ANTI-FRICTION ADDITIVES IN PERFORMANCE IMPROVEMENT OF FRICTION PAIRS
}

\author{
Irina Belinskaia, Alexander Evseev \\ Saint-Petersburg State Agrarian University, Russia \\ belinska@yandex.ru,post44@inbox.ru
}

\begin{abstract}
An effective way to increase serviceability of the crankshaft engine is the use in lubricating antifriction additives. This allows to significantly extend the service life of the engine and to reduce the financial cost of its maintenance. One of the varieties of anti-friction additives - friction geomodifiers - is the additive "Energy and resources saving" (ERS). The main objective of the use of anti-friction additives ERS optimization of clearances in the pair carried out by building a protective layer. Raw materials for the manufacture of ERS are one of the varieties of silicates - layered silicates - serpentine. It is formed under the influence of hydrothermal solutions on ultramafic olivine rocks. Base of such rocks is silica oxide radical in the form of a tetrahedron. The accumulated theoretical and practical experimental base suggests that using lubricants with the addition of serpentine-type friction geomodifiers significantly increases the serviceability of pairing, wear resistance of mating parts is increased 2-4 times. At the same time the loss of energy to overcome friction is reduced 1.5 times, while the roughness of metal friction surfaces - several times. The aim of the study is to determine the influence of ERS additive concentration in motor oil on the serviceability of the engine's crankshaft bearing in moments of operation, when the effect of hydrodynamic lubrication is reduced. The conducted experiments show that the concentration of anti-friction ERS additive in modified oils significantly influences the process of friction of the surfaces. Positive effect of oil modification occurs at concentrations of more than $0.5 \%$. Increasing the concentration of additive above $2 \%$ leads to deterioration in the service life of friction pairs. The minimum value of the coefficient of friction and roughness parameters of the friction surfaces for the friction pair "Steel 40X - Alloy ASM" correspond to concentrations of 1.8 to $1.9 \%$.
\end{abstract}

Keywords: geomodifiers, friction, sliding bearing, serviceability of crankshaft, coefficient of friction.

\section{Introduction}

Operation of any technical system is impossible without the friction process. Over time, as a result of friction, the efficiency of internal combustion engines decreases, its power decreases, the consumption of fuel and lubricants increases, the leakage of explosive products through seals increases, the economy, reliability and durability of the car decreases, its dynamic and acoustic characteristics deteriorate.

Rolling and sliding supports, transmission mechanisms, braking devices, sealing elements, friction units of transport equipment will be able to function successfully only with the use of special materials, lubricants, coatings - antifriction additives.

Repair-and-renewal preparations on the composition, physicochemical processes of their interaction with friction surfaces, the properties of the obtained coatings (protective films), as well as the mechanism of operation in the course of further operation is divided into three main groups: remetallized, polymerized drugs and geomodifiers.

The fundamental difference between the geomodifiers of friction lies in the fact that in a tribological system a substance that triggers self-organizing processes is introduced. Other types of repair preparations affect the separation of rubbing surfaces by the third body (soft metals, long hydrocarbon chains, synthesized film), but geomodifiers help the tribotechnical system to determine, which the surface structure should be, the height of the modified structure, roughness, waviness, etc.

The main advantages of friction geomodifiers are:

- ability to create dynamic protective films formed by fine products and the geomodifier itself in the form of a quasi-liquefied layer, which allows you to repeatedly reduce the wear rate of friction units;

- displacement of characteristics closer to hydrodynamic friction and, consequently, reduction of mechanical losses;

- chemical, electrical neutrality and environmental friendliness of the natural product;

- unchanged range of modes of operation of the friction node of the modified layer is maintained until the thermo-cyclic fatigue destruction [1]. 
Professor Kragelsky I. V. through the analysis of the research results of friction processes has formulated three consecutive and interrelated stages of the process of friction: interaction between the surfaces with regard to the effects of environment, microstructure changes of the surface layers as a result of interaction, the fracture surfaces as a consequence of the previous two stages [2]. These findings are the basis for experimental studies of all types of geomodifiers.

\section{Materials and methods}

The action of repair and recovery compositions is containing mineral additives of geological origin, based on the unique properties of serpentine powder. The action of serpentines based on silica oxide radical [SiO4]4 - in the form of a tetrahedron. Theoretical study of geomodifiers application on the basis of serpantine began in the 1990s by the British scientist Kaeker J. [3]. Earlier studies [2] show that using lubricants with the addition of friction geomodifiers -type serpentines can greatly increase the efficiency of the mates. At the same time the loss of energy to overcome friction can be significantly reduced, and the wear resistance of the mating parts increases by 2-4 times, the roughness of metal friction surfaces may also be reduced by several times [2].

Large-scale studies of the use of serpentine-based geomodifiers were conducted at Tsinghua University [5]5]. Laboratory tests of application of a covering on a cylinder liner of the diesel $16 \mathrm{~V} 280 \mathrm{Z}$ were carried out. The result revealed that fine mixture of serpentine contributes to formation of emission of particles, pyrolysis of the components of the lubricant, tribocatalytic carbonization and formation of the hard carbon-containing compounds from oils; confirmed by the low coefficients of friction and thermal conductivity, high strength and corrosion resistance of geomodifiers [5].

Geomodifiers from mineral mixtures of serpentine group have wide practical application: in Russia - SUPROTEC, MEGAFORCE, Forsan nanoceramics, REAGENT 2000, Supro, in Japan Metaryzer, in Germany - Revitec, in Finland - RVS-tec, in Sweden - RESTAL.

One of the modern geomodifier kind based on serpentine is the additive ERS (energy and resources saving). The composition of ERS consists of a fine mixture of minerals with the particle size not more than $1 \mu \mathrm{m}$, it contains working supplement, deoxidizers and surface catalysts. The composition is blended in base oil with a thickening agent, which is compatible with any regular oils and greases. It does not enter into any chemical reaction with commodity oils, grease and additives. ERS works only with metal, its surface friction and only if there is energy released upon the contact of the friction surfaces.

Features of geomodifier ERS:

- freezing point $-50{ }^{\circ} \mathrm{C}$;

- does not affect the physico-technical properties of the oil;

- does not contain any of the known substances and additives that reduce friction, such as talc, graphite, molybdenite or industrial diamonds;

- compatible with any lubricant;

- allows to completely prevent the contact metal-metal, synthesizing at the contact layer of the cermet, a material with unique physical-mechanical properties;

- mechanisms without wear tolerate cold start and high loads;

- restores and strengthens the surface of friction;

- stops the wear of friction pairs;

- allows almost completely eliminate adhesion and diffusion wear in kinematic pairs of friction;

- reduces vibration and noise during operation;

- prevents electrochemical corrosion;

- reduces the requirements to the quality of fuel and engine oil;

- in emergency situations, when adherence of the movement (the engine speed must not exceed $2000 \mathrm{rpm}$ ), possible temporary work of the engine without oil (up to $1000 \mathrm{~km}$ ).

Scientists from SPbSAU conducted operational testing of the geomodifiers [6]. The result revealed that when adding geomodifiers ERS in the lubricant a wear-resistant layer is formed, which has the following features:

- smooth surface, on which the oil film works very fine; 
- the same linear thermal expansion as metal, during heating and cooling it is not destroyed and does not flake;

- the thickness required for wear compensation and optimization of gaps in friction pairs.

Technical and economic feasibility of applying the technology of ERS is that it allows to:

- renew the values of operating parameters of used machines, units and mechanisms in their normal operation;

- support the restored operating parameter values throughout the life of the equipment;

- reduce the operating costs;

- increase the service life of motor oils by 2 times, and transmission oils - 4 times;

- decrease the consumption of motor fuel by $8-12 \%$ [6];

- reduce energy consumption by $8-15 \%$ [6].

In further studies the tests of the possibilities of ERS geomodifiers were conducted. It allows to determine the influence of ERS additive concentration in motor oil on the operability of the engine crankshaft bearing in moments of operation, when the effect of hydrodynamic lubrication is reduced.

For the laboratory tests on the wear and friction the friction machine CMT1 is used. The machine provides several speeds of rotation and the ability to measure the friction torque. The main technical data of the machine:

- $\quad$ speed of the lower sample shaft-from 75 to 1500 min-1;

- maximum allowable moment for samples of friction "Roller-Block" $-10 \mathrm{~N} \cdot \mathrm{m}$;

- measuring ranges of the efforts of the samples from 200 to $2000 \mathrm{~N}$.

The studies were carried out on the machine friction scheme "block" (element of bushing) "roller" (element of crankshaft). Based on the analysis of the friction pair the crankshaft bearing for the research as a part of the "roller" was applied to the samples that were made according to the technological standards for the manufacture of crankshafts with subsequent thermal treatment. The surface hardness is $52 \ldots 62 \mathrm{HRC}$, roughness of the surface after finish treatment amounted $0.32 \ldots 0.16$ $\mu \mathrm{m}$. As part of the "block" fragments of bearings were used, the working surface of which is antifriction alloy ASM.

Lubrication of the friction surfaces was carried out by wetting with oil poured into the test cell. The study was carried out on the oil M8 group, the viscosity of SAE 20.

In the process of the research the following parameters were measured:

1. The friction torque. The measurement was carried out by using a digital voltmeter connected to the output voltage of the measuring transducer of the friction machine with the subsequent calculation of the friction torque with an accuracy of $0.1 \mathrm{Nm}$.

2. The temperature in the friction zone. The measurement was carried out the control and measuring complex, consisting of the thermocouple "chromel-drops" and the meter. Measurement accuracy of $0.1^{\circ} \mathrm{C}$.

3. The area of actual contact in the mate. The area was determined by caliper measurement of the contact boundaries and calculation of the area in $\mathrm{mm}^{2}$.

4. The roughness parameters of the working surface of the roller. The roughness parameters determined by the device MITUTOYO "Surftest SJ-301".

The number of repetitions of the experiments was chosen to provide accurate results with a confidence probability of 0.95 with a relative error of $3 \%$.

\section{Results and discussion}

Fig. 1 shows the comparative characteristics of the change of the coefficient of friction pair "Steel 40CR - Alloy ASM", when working on the M8V oil without additives and with addition of $1.5 \%$ ERS, depending on the contour of the pressure.

The graph shows that additive ERS major impact at high contour pressures, when the friction pair does not work under oil lubrication without additives. 
To estimate the running-properties the antifriction additive test was made in two steps. At first, the initial roughness of the roller corresponded to the polished surface of the crankshaft journals - Ra of $0.18-0.22 \mu \mathrm{m}$, and the second - the roughness of the roller corresponded to the abrasive surface of the crankshaft journals - Ra of $0.45 \mu \mathrm{m}$.

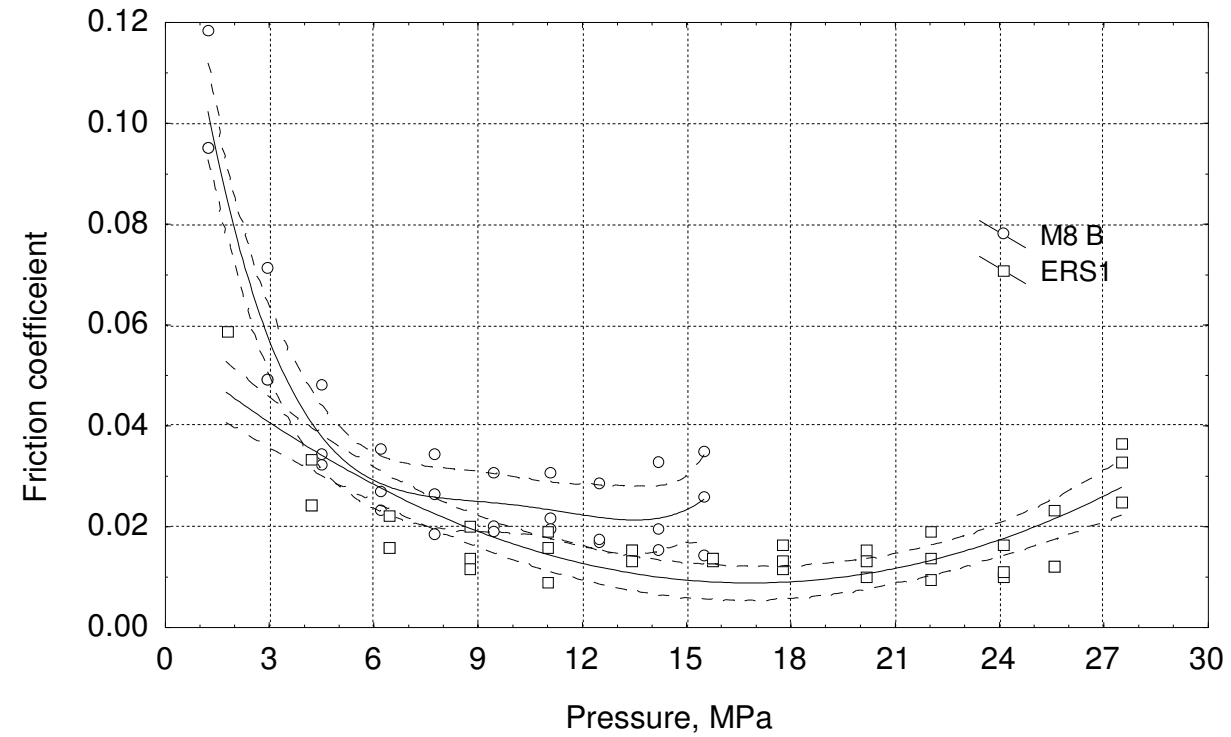

Fig. 1. Dependence of coefficient of friction in friction pair "Steel 40CR - Alloy ASM" when working on M8Voil without additives and with addition of $1.5 \% \mathrm{ERS}$

Fig. 2 shows the change in the parameters of surface roughness of the shaft at different initial roughness depending on increase in contour pressure during work of pairing using the oil without additive and with the additive ERS.
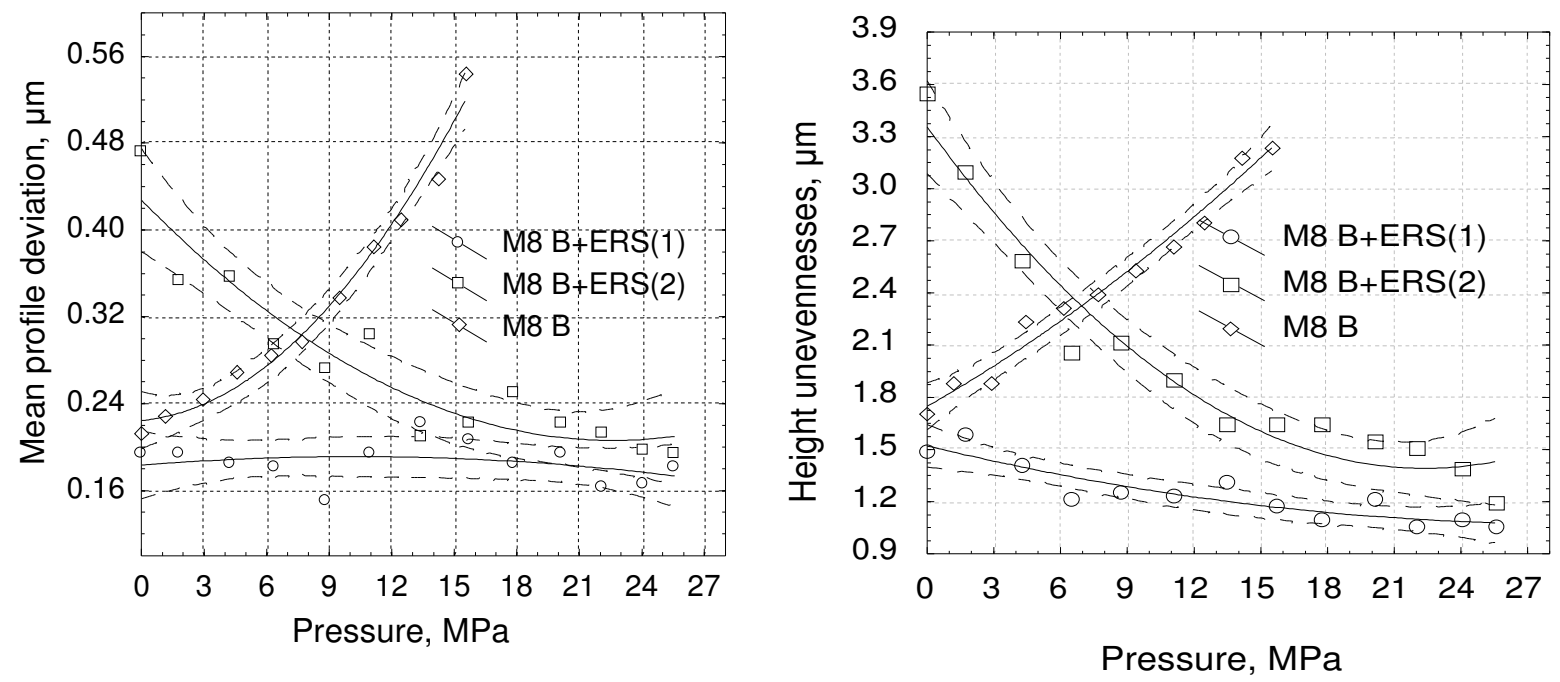

Fig. 2. Dependence of mean profile deviation $(R a)$ and height unevennesses of profile at 10 ponts $(\boldsymbol{R z})$ on countour pressure during process of run-in. M8B+ERS(1) - oil M8B with ERS addition and initial shaft roughness $0.2 \mu \mathrm{m}$; M8B+ERS(1) - oil M8B with ERS addition and initial shaft roughness $0.45 \mu \mathrm{m}$

Changes in all of high-rise parameters of the roughness of the roller surface show that, when working on oil without additives, the equilibrium roughness considerably varies with the pressure. Moreover, the values of all parameters increase.

The change of the friction coefficient depending on the testing time and increasing load for different values of the ERS concentration in the lubricating oil is shown in Fig. 3. 
From the presented dependences it follows that the processes can be divided into two groups. The first group includes processes for the additive concentration in the range from 0 to $0.5 \%$. With this concentration, the friction processes do not differ from the process of friction without additives. The diffusion coefficient of friction is within the limits of error of the tests; significant changes in the process of changing the friction coefficient are not observed.

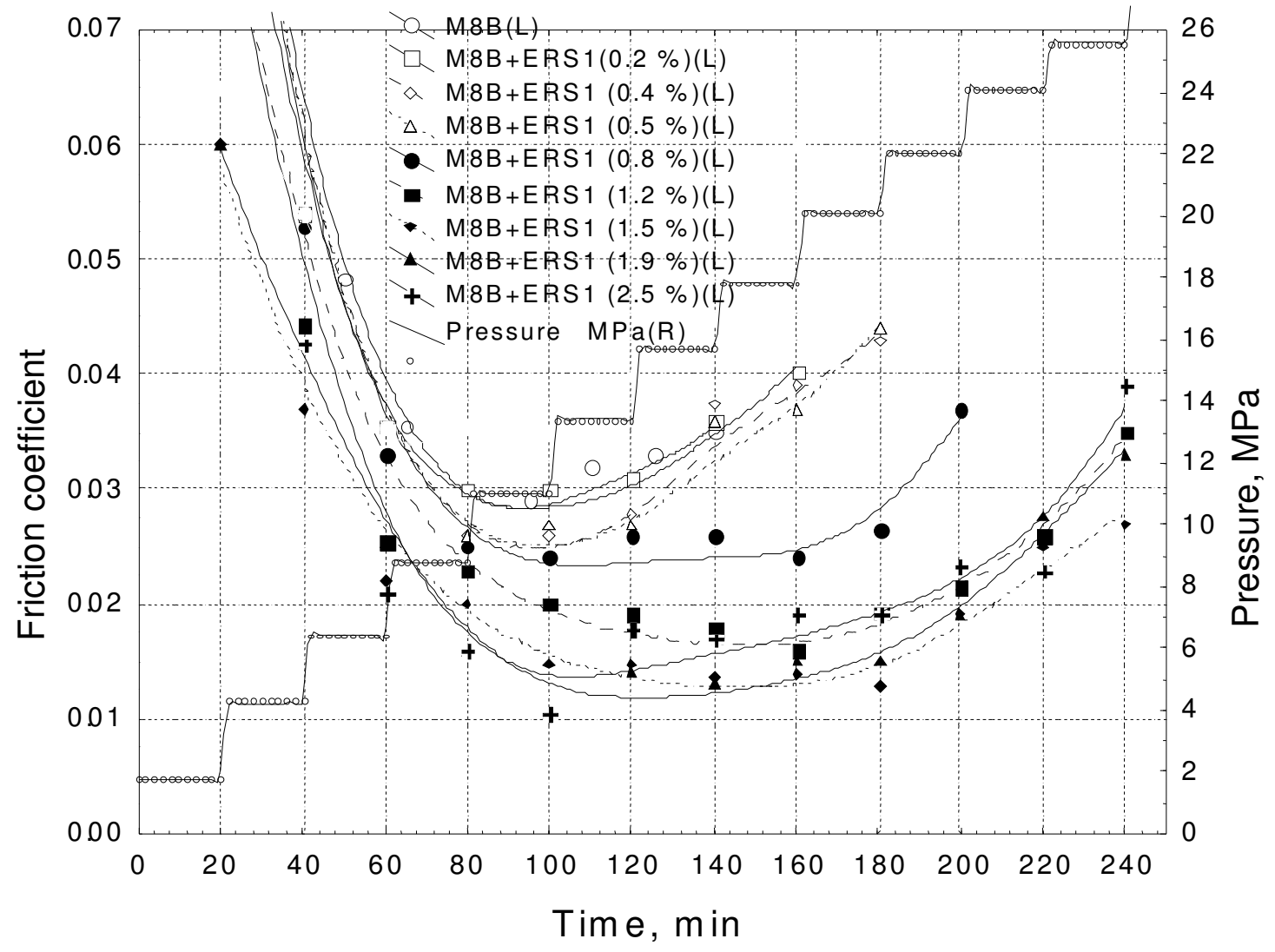

\section{Fig. 3. Change of friction coefficient depending on testing time and increasing load when varying ERS concentration in lubricating oil}

When the concentration is more than $0.5 \%$, the friction process changes and the friction coefficient decreases. This trend continues up to concentrations of $1.2 \%$.

The second group includes processes for the additive concentration in the range of $1.2 \%$ to $2.5 \%$. In this case, there is a significant difference to the process for the oil without additive.

Run-in processes of the rubbing surfaces for the two groups differ. For processes of the first group in the initial period the coefficient of friction is slightly higher than for the processes of the second group. This is because the additive at a concentration of $0.2 \%$ to $0.5 \%$ has little influence on the process of friction and wear, and dispersion of initial values has a small difference of initial roughness parameters of the samples.

For concentrations in the range of 1.2 to $2.5 \%$ the effect of additives has been observed since the beginning of the tests. The coefficient of friction for the first 20 minutes fell to an average of $40 \%$. This decrease continues throughout the whole running time. In general the run-in period is 1.5 hours.

Additive ERS generates an equilibrium surface roughness that reduces the intensity of wear of rubbing surfaces. For the friction pair "Steel 40CR - Alloy ASM" arithmetic mean deviation of the profile $\mathrm{Ra}$ reduced by 2 times (depending on pressure), the height of roughness at ten points $\mathrm{Rz}$ - up to 3 times (depending on pressure), the maximum height of the irregularities Rt - to 2 times (depending on pressure). 


\section{Conclusions}

The studies show that the concentration of anti-friction ERS additives in the modification of oils significantly influences the process of friction of the friction surfaces:

- the effect of oil modification occurs at a concentration of more than $0.5 \%$, increasing the concentration of additives above $2 \%$ leads to a deterioration in efficiency of friction pairs;

- the equilibrium roughness varies considerably and depends on the pressure; the parameters (deviation of the profile and height of irregularities) are decreased;

- the average friction coefficient is reduced by $40 \%$;

- the minimum value for the friction coefficient and roughness parameters of the friction surfaces for the friction pair "Steel 40CR - alloy ASM" correspond to the concentration of $1.8-1.9 \%$.

\section{References}

[1] Garkunov D.N. Tribology. Moscow: MAA, 2001. 616 p.

[2] Kragelskiy I.V. fundamentals of friction and wear calculations. Moskow: engineering. 1977. 350 p. (In Russian).

[3] Kaeker J.J. Three dimensional Elastic Bodies in Rolling Contact. Dordrecht; Boston: Kluwer Academic Publishers, 1990. 314 p.

[4] Dunaev A.V., Sharifullin S.N. Modernization of worn-out equipment with tribo solutions. Kazan: KU, 2013. 272 p. (In Russian).

[5] Yuansheng J., Shenghua L. Superlubricity of in situ generated protective layer on worn metal surfaces in presence of $\mathrm{Mg}_{6} \mathrm{Si}_{4} \mathrm{O}_{10}(\mathrm{OH})_{8}$ / Superlubricity. Edited by Ali Erdemir / Argonne National Laboratory.- Argonne, USA; and Jean-Michel Martin / Ecole Centrale de Lyon.-Lyon, France. Elsevier B.V., 2007. pp. 445-469.

[6] Skovorodin V.Y., Evseev A.S., Jamilov M.K. A study of the effectiveness of anti-friction ERS additives to increase efficiency crankshaft bearings of automotive engines. Proceedings of the International Academy of agrarian education. Vol. 6. SPbSAU. 2013. pp. 68-72. 